\title{
Spreadsheet modeling for research and teaching: Programming without programming
}

\author{
CHARLES E. COLLYER \\ University of Rhode Island, Kingston, Rhode Island
}

\begin{abstract}
Spreadsheets can be used to focus academic research and teaching on theoretical models. Examples of models from learning, social psychology, and perception are presented to illustrate how spreadsheet techniques work. Two strengths of this approach are emphasized: (1) Spreadsheets provide a relatively user-friendly alternative to some kinds of instructional and research programming; and (2) the linked tables and graphs of modern spreadsheets provide a powerful display medium and a fast way to examine the behavior of models as parameters change. I suggest some models for which spreadsheets may be appropriate.
\end{abstract}

A spreadsheet is a type of software for managing and processing information in a large row-by-column matrix of cells. The cell entries can be numbers, text (either visible or hidden as supplementary notes), or formulas. Operations such as copying, merging files, and making charts from tabled data can be carried out by keyboard commands or by a pointing device such as a mouse. Procedures called macros can be defined that carry out a series of operations automatically. Some good spreadsheet programs are Microsoft's Excel, Borland's Quattro Pro, and Lotus's 1-2-3. I will use Excel examples and notation here.

A number of articles on uses of spreadsheets have appeared in this journal recently. Hewett (1988) discussed ways of using spreadsheets to enhance professional productivity. For example, a schedule in the form of a Gantt chart can be constructed which allows the user to visualize the various tasks and committments that should receive attention during specific time periods. Rogers (1989) described spreadsheet demonstrations that were helpful in teaching statistical concepts such as correlation and analysis of variance. Hewett (1985) and Halff (1987) presented excellent discussions of spreadsheet models that simulate specific sensory and neural processes.

In the present paper, I argue that spreadsheets are a good vehicle for building theoretical models and teaching about them, and perhaps for extending model-oriented thinking to a larger number of people in psychology. The case for mathematical models in psychology has been made before; for example, Bjork (1973) and Estes (1975) presented eloquently persuasive introductions. The type of mathematical modeling advocated by Bjork and by Estes has enjoyed mixed success. Mathematical models have become the standard medium for expressing theoretical

Preparation of this article was spported in part by Grant NSS2-S07RR07086-14 to the University of Rhode Island from the National Center for Research Resources. Thanks are due Susan A. Brady, Hilary A. Broadbent, and Russell M. Church for helpful suggestions. Correspondence should be addressed to Charles E. Collyer, Department of Psychology, University of Rhode Island, Kingston, RI 02881. ideas in some research fields in psychology. The general theory journal Psychological Review, for example, is an important place to look for new mathematical models. However, an explicit modeling approach has been neglected or rejected by many psychologists. Some psychologists have complained to me that although modeling is clearly valuable, the effort required to learn and work with models is daunting. Perhaps reducing the perceived effort would result in more modeling. Spreadsheets can make mathematical models both more accessible for novices and easier to work with for more experienced users. I hope that this article will encourage some of my colleagues to try modeling through this medium.

\section{Constructing Spreadsheets}

A spreadsheet can be used as a temporary scratchpad by one person, much as a calculator would be used. In this case, the structure of the spreadsheet may not be very important. However, some spreadsheets are intended to be used repeatedly, or must be intelligible to persons other than their author. Such spreadsheets should be considered true computer programs, and their design deserves the careful consideration that would be given to any serious writing or presentation project. Nevison (1990) has suggested a number of guidelines for authors of businessoriented spreadsheets, which are adapted here with an academic slant.

Nevison (1990) recommends that a spreadsheet contain three functionally different areas: the introduction, the initial data area, and the model area. The example spreadsheets in this article all have this three-part structure.

The introduction should identify the spreadsheet and provide useful orienting information. First, title lines should give the file name of the spreadsheet and a more complete title indicating its topic. The purpose of the spreadsheet can be explained in a short paragraph. Brief directions on how to use the spreadsheet should be given, especially if there will be no accompanying documentation for users. References to articles or books can be included if appropriate. 
The initial data area should be a compact region of the spreadsheet where parameters of the model are identified and entered. (Perhaps the term parameters area would be more in keeping with conventional modeling language.) Brief notes about the effects of manipulating specific parameters can be included. The cells of the initial data area that contain parameter values will be accessed by other cells in the following model area to drive the model's behavior. If appropriate, summary measures of the model's behavior can be returned to the initial data area for convenient inspection. Thus, the initial data area can be thought of as the user's "control panel" for both manipulating and monitoring the output of the full model.

The model area is a region of the spreadsheet containing formulas that generate data according to the equations that define the theoretical model of interest. Parameters of the theoretical model should be fed to these formulas from the initial data area. Summary measures of the model's output, such as a measure of goodness-of-fit (of the model's output to a set of empirical data values, for example), can be fed back to a cell near the parameters, so that the overall effects of parameter changes can be seen conveniently at the user's control panel.

A spreadsheet should be checked carefully for accuracy. Its output under standard parameter settings should be examined to ensure that known conditions in fact generate the expected results. Some of the software's own features can be helpful in tracking down errors. In Excel, the Options/Display/Formula command can be used to produce a version of the worksheet in which all formulas (rather than their output values) are displayed. The accuracy of formula structures and cell references is easier to check with this version than with the display of numerical values.

Let us consider first how a spreadsheet might be used to illustrate a well-known model of learning. Once we have this example in hand, I will note two significant strengths of spreadsheet models and outline two more modeling examples.

\section{Learning: The Rescorla-Wagner Model}

The Rescorla-Wagner model (Rescorla \& Wagner, 1972) is a theory of classical conditioning, the kind of learning in which an animal or a person experiences a correlation between two events (in Pavlov's famous case, the bell and food) and eventually demonstrates a change in behavior (such as salivation to the bell) which reveals that the correlation has been learned. The basic idea of the Rescorla-Wagner (R-W) model is that the amount learned from a pairing of the two events depends on how surprising the second one is. The "amount learned" adds to associative strength, with the increment being larger when the second event is more surprising.

The model proposes that associative strength grows from one learning trial to the next, according to this equation:

$$
V_{n}=V_{n-1}+a \cdot b\left(L-V_{n-1}\right),
$$

where $V_{n}$ is the associative strength on the current trial, $V_{n-1}$ is the associative strength accrued up to the pre- ceding trial, $L$ is the maximum possible value of associative strength, and $a$ and $b$ are measures of the salience to the organism of the first and second events, respectively. Equation 1 expresses the idea that associative strength on a given trial is composed of its value on the previous trial, plus an increment that is proportional to $\left(L-V_{n-1}\right)$. This "surprisingness" term, $\left(L-V_{n-1}\right)$, represents how much more learning about the correlation is possible. (Equation 1 is one expression of the $R-W$ model. Several variations on the model have been proposed, including those of Sutton \& Barto, 1987, and Gluck \& Bower, 1988. It has been pointed out by these investigators and others that Hebbian learning rules in connectionist models are expressed by equations similar to the R-W model.)

Figure 1 shows a spreadsheet file (in Excel, a worksheet) called RESWAG.XLS. (The filename extension .XLS stands for 'Excel sheet.') Rows 1-10 give introductory information, including the basic equation of the model. Rows 12-16 identify the basic terms of the model, which include the independent variable, $n$, and the model parameters $a, b$, and $L$.

Two specific $V_{n}$ functions are shown in the lower part of the spreadsheet (rows 18-47). On the left (columns A and $B$ ) is a fixed example of the function, generated with the specific parameter values $a=0.3, b=0.5$, and $L=1.0$. Thus, the formula in cell B23 was entered as follows:

$$
=\mathrm{B} 22+(.3) *(.5) *(1-\mathrm{B} 22) .
$$

(In Excel, formulas begin with the " =" sign. A " +" sign will also work.) The entries in cells B24-B47 were made by "filling down" from cell B23 with one command (Edit/Fill Down). In this use of the Fill Down operation, Equation 2 was copied into each cell, but with relative reference to cell B22 such that the formula in cell $\mathrm{B}(n)$ appropriately operates on the value in cell $\mathrm{B}(n-1)$. Absolute reference is an alternative way of obtaining values from other cells; it is illustrated in the next paragraph.

On the right side of the spreadsheet (columns $E$ and $F$ ) is a "what-if" example, in which the values of $a, b$, and $L$ are always taken from the entries in cells F14, F15, and F16. The formula entered into cell F23 in this example was:

$$
=\mathrm{F} 22+(\$ \mathrm{~F} \$ 14) *(\$ F \$ 15) *(\$ F \$ 16-F 22) .
$$

Notice that, for the purpose of cell reference within the spreadsheet, the model terms $a, b$, and $L$ have been replaced by absolute cell addresses, which can be recognized by the dollar signs preceding the column letter and row number. Absolute addresses are not adjusted by Excel during Fill operations, but relative references, such as the reference to cell F22, are adjusted. A set of appropriately adjusted formulas based on Equation 3 was entered into cells F24-F47 with the Edit/Fill Down command as before. Because the references to cells F14, F15, and $\mathrm{F} 16$ were absolute, the current values of $a, b$, and $L$ are used as constants throughout this set of formulas. However, because the reference to cell F22 was relative, 


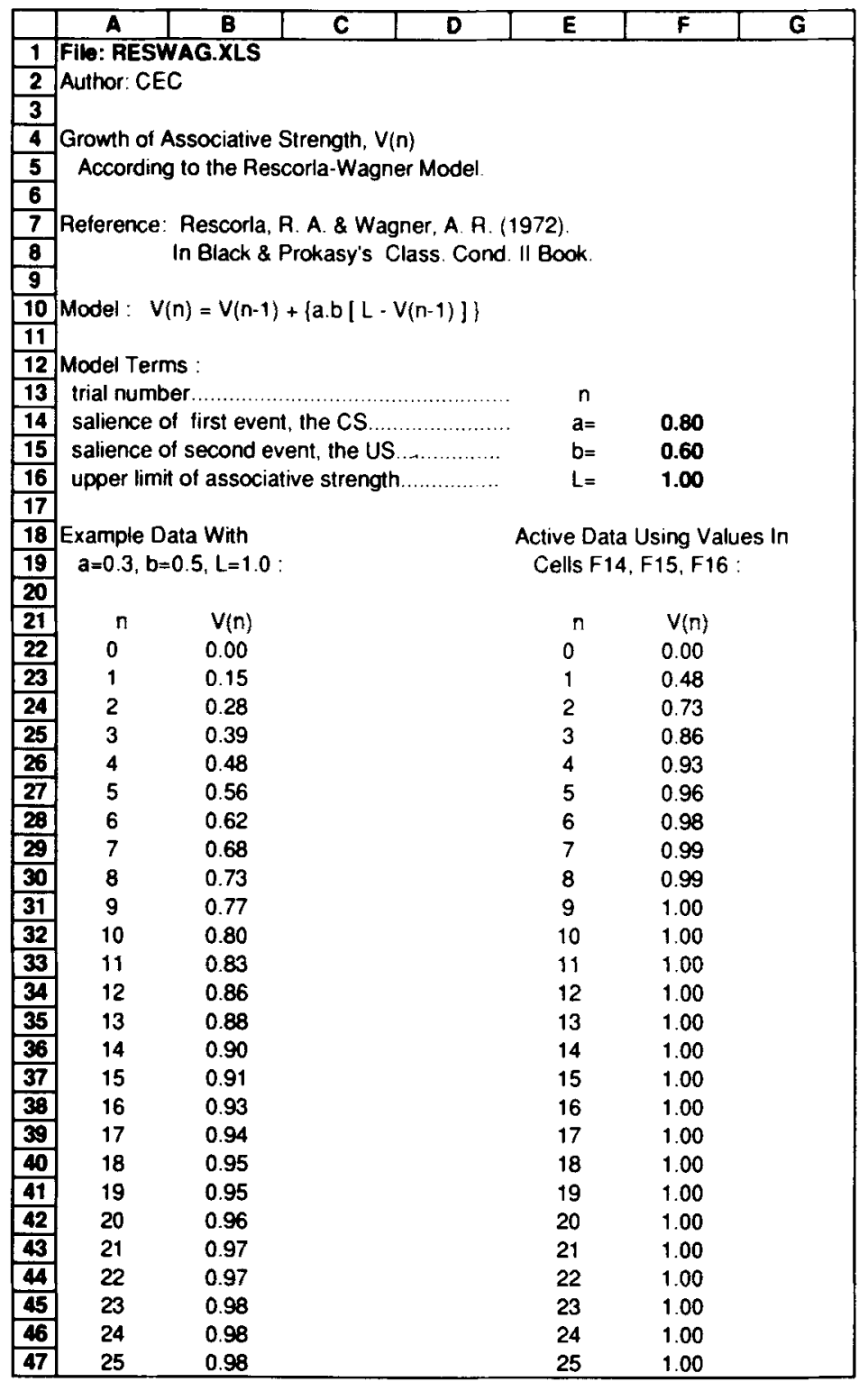

Figure 1. Spreadsheet RESWAG.XIS, illustrating the Rescorla-Wagner model. Entering new parameter values in any of the cells F14, F15, and F16 would cause the predicted series of associative strengths in cells $\mathrm{F22}-\mathrm{F} 47$ to be recalculated.

it was adjusted (from F22 to F23, F24, and so on) as the formulas were filled (from F23 to F24, F25, and so on).

With this set of formulas in place, typing a new value into any of the three parameter cells F14, F15, or F16 would cause the "what-if" values of $V_{n}$ in the lower right portion of the spreadsheet to be recalculated accordingly.

The effects of playing "what-if" with the RescorlaWagner model can be visualized by constructing a graph (in Excel, a chart) of the data in the lower right portion of the spreadsheet. Figure 2 shows such a graph. An Excel command, Windows/Arrange All, allows the spreadsheet and the graph to be displayed on the monitor screen side by side. When a change is made in one of the three parameters in the spreadsheet, two things happen: the data values in the formula-driven part of the spreadsheet are recalculated, and the graph is replotted using the new values. The graph remains hot-linked to the data displayed on the spreadsheet, and any number of further parameter variations can be explored.

Here is an example of one such variation. Figure 3 shows the same chart as Figure 2, except that the value of the parameter $a$ in cell F14 of the worksheet has been changed from 0.2 to 0.8 , leaving $b=0.6$ and $L=1$ as before. The chart's change in appearance from Figure 2 to Figure 3 took only a few seconds from the time of entry of the new value in cell F14. Such an increase in the 
RESCORLAWIGER WOOEL WTHI $=2,20: 6, \mathrm{~L}, \mathrm{~L} 1$



Figure 2. Chart RESWAG.XLC, displaying data from the active model area of spreadsheet RESWAG.XLS. In this example, the parameter values were $a=.2$, $b=.6$, and $L=1$.

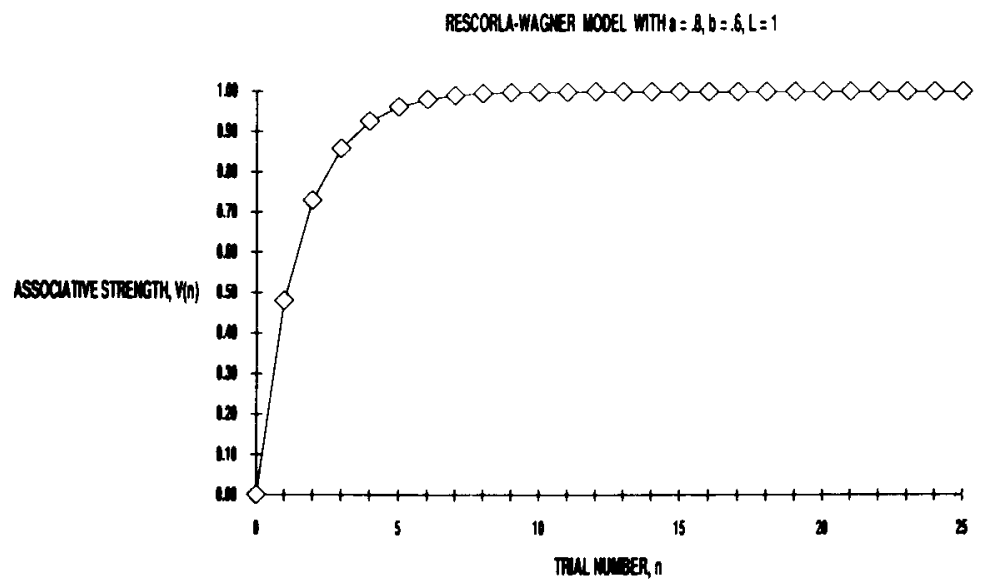

Figure 3. Chart RESWAG.XLC, with parameter values $a=.8, b=.6$, and $L=1$.

value of $a$ corresponds to an increase in the salience of the conditioned stimulus, which might be brought about by increasing its intensity, for example.

\section{Two Attractions of Spreadsheet Models}

Two features of spreadsheet models are well illustrated by the R-W example. First, a spreadsheet model amounts to programming without actually writing a program. That is, many of the operations carried out by the spreadsheet model could have been programmed in FORTRAN, BASIC, C, or Pascal. However, apart from getting a formula's syntax right, little was done in constructing the spreadsheet that would remind a frustrated former programmer of traditional program coding. Spreadsheets could be used in some areas of research where more laborintensive programming for simple analysis or simulation is now the norm. For example, Monte Carlo simulations and schedules for random assignment or sampling can use the random number function available in almost all spreadsheet programs. Spreadsheets may also be attractive to teachers who would prefer not to write their own instructional software, but whose material lends itself to this format.

Second, a spreadsheet built on formula entries and hotlinked to a chart display has considerable value for discovery-oriented learning and instruction. Quickly seeing the effects of a parameter change on a model's output probably helps most people understand the model.

\section{Interactional Model of Stress}

The interactional model (e.g., Cox, 1978; Lazarus, 1976) is a plausible conceptualization of how factors inside and outside a person combine to determine stress. The model can be expressed by the equation

$$
S=B+M(D-C) .
$$

In this model, the stress $(S)$ experienced by a person is related to the difference between environmental demands $(D)$ and coping abilities $(C)$, multiplied by a motivational factor $(M)$ representing how much the person cares about 


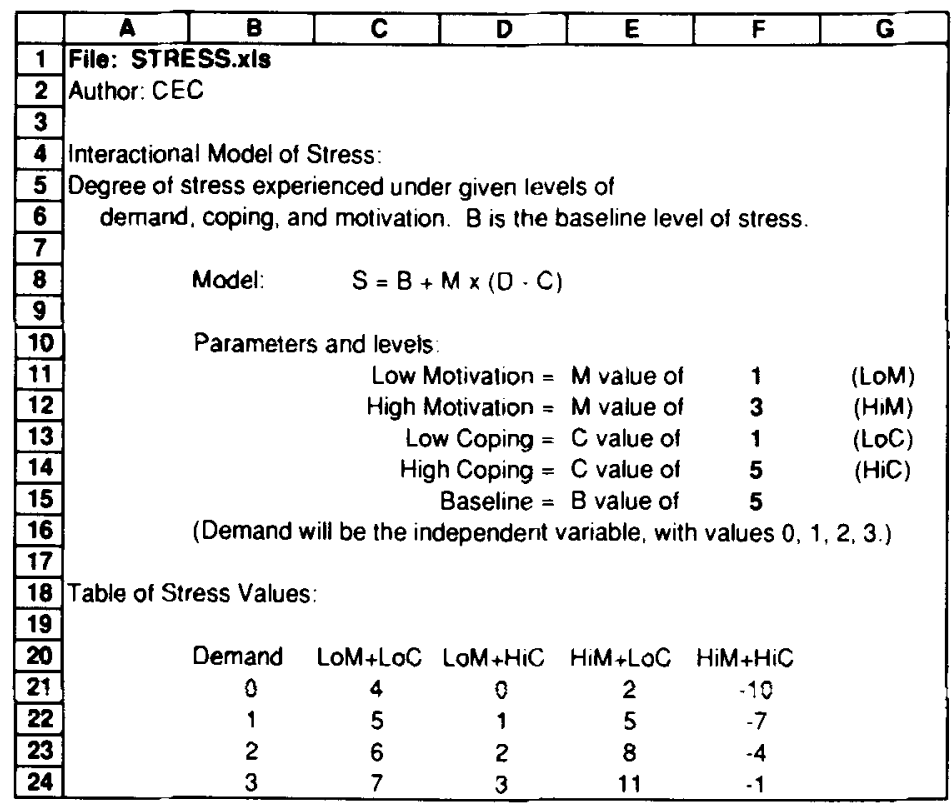

Figure 4. Spreadsheet STRESS.XLS, illustrating the interactional model of stress. Entering new parameter values in any of the cells F11-F15 would cause the predicted pattern of experienced stress levels in cells C21-F24 to be recalculated.

the outcome of the situation. The term $B$ represents a baseline level of stress, which is independent of the other factors. To work with the model, these variables are given arbitrary numerical values, as in Figures 4 and 5.

Figure 4 shows a spreadsheet for the interactional model, and Figure 5 shows an Excel bar chart fed by the stress values in cells C21-F24 of the spreadsheet. A number of features of these linked displays are noteworthy.

First, the goal of constructing a relatively simple chart dictated some simplification of the spreadsheet model. Only two levels of motivation (low and high) and coping (low and high) were examined, and the baseline was held constant (one value of 5). Excel could calculate the model for hundreds of levels of the variables, but in this case, comprehensibility took precedence over power.

Second, the table of stress values was constructed with the leftmost column (demand) as the main independent variable (and abscissa of the linked graph), and the other columns all representing the single dependent variable stress, but under different conditions of motivation and coping. If the sequence of commands given in the Excel manual for constructing scatter charts is followed, the result is a conventional (to an academic's eyes) arrangement of axes and curve parameters.

Third, there is linkage between the parameter values in cells F11-F15 (initial data area) and the table of stress

\section{INTERACTIONAL MODEL OF STRESS}

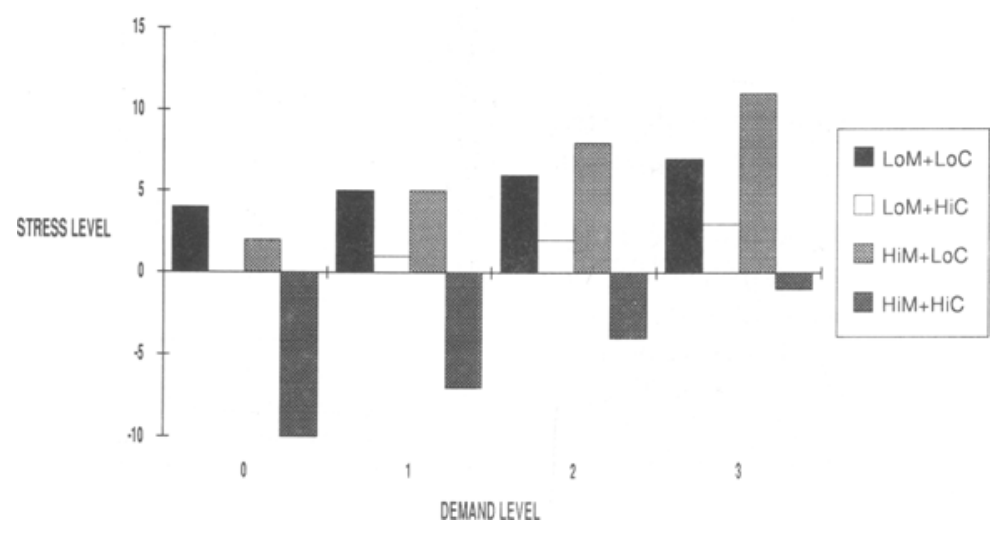

Figure 5. Chart STRESS.XLC, generated from the table of stress levels in spreadsheet STRESS.XLS. 
values (model area). Typing a new parameter value in one of these cells changes some or all of the stress values. For example, the formula in cell $\mathrm{C} 21$ is

$$
=\$ F \$ 15+\$ F \$ 11 *(\mathrm{~B} 21-\$ F \$ 13) \text {, }
$$

which corresponds to

baseline + low motivation $\times$ (high demand - high coping),

with the current values

$$
5+1 \times(0-1)=4 \text {. }
$$

Recall that \$ signs are used for absolute referencing. Absolute referencing is generally used when a parameter value must be held constant throughout a series of calculations along rows or columns, whereas relative referencing is used when an independent variable changes systematically throughout such a series.

The fourth feature is the linkage between the table and the chart. Changing a parameter results in the recalculation of the tabled stress values, which then automatically update the graph. "What-if' experiments, graphically displayed, can be done in a few seconds to explore the model's predictions of stress levels under changed conditions of demand, motivation, and coping ability.

One way of understanding the "interactional" property of the model is to note that the effect of demand on stress may be either small (under low motivation) or large (under high motivation). The same may be said of the potential effects of coping. The spreadsheet model shows clearly how this multiplicative type of interaction works.

\section{Comparing Models With Each Other: \\ Fechner's Law and Stevens's Law}

As a last example, let us consider two classic models from psychophysics. Fechner (1860/1966) proposed that the subjective magnitude of a sensation, $S$, is a logarithmic function of the stimulus intensity, $I$ :

$$
S=k \log (I),
$$

where $k$ is a scaling constant and it is understood that $I$ has been corrected for the person's absolute threshold. Stevens $(1957,1961)$ argued instead that sensation magnitude is a power function of intensity:

$$
S=k^{\prime} I^{n},
$$

where $k^{\prime}$ is another scaling constant and $n$ is the exponent or power to which $I$ is raised for a particular sensory modality.

Both versions of the "psychophysical law" relating $S$ and $I$ are familiar to students of general psychology and perception. Indeed, there is an extensive literature on the scaling of subjective magnitude, which has influenced applied measurement in several areas. Recently Krueger (1989) has attempted to reconcile the Fechner and Stevens laws. We shall borrow just one of the ideas in Krueger's extensive analysis in order to develop another example of spreadsheet modeling.

One of Krueger's (1989) observations was that data generated by a logarithmic process (Fechner) can be quite well fit by a power function (Stevens) that has a low exponent and a suitably chosen scaling constant. Opinions may differ on the proper conclusions to be drawn from this observation; however, the properties of the examples used by Krueger to make his point can easily be explored by setting up a spreadsheet such as the one in Figure 6, linked to a chart such as the one in Figure 7.

The cells B24-D35 of the spreadsheet in Figure 6 provide the data for the chart. The values shown are from one of Krueger's (1989) examples (p. 254, his Figure 1A), in which subjective magnitudes simulated under the two laws were shown to be very similar under certain conditions. These data values were generated by formulas corresponding to Equations 5 and 6, using parameters specified in rows 16 and 17 of the worksheet. To quantify the degree of similarity of the two laws, the spreadsheet includes columns for the difference and squared difference of the two functions at each intensity. The sum of the squared differences (cell F37, whose value is also copied back to cell $\mathrm{C20}$ ) gives an overall measure

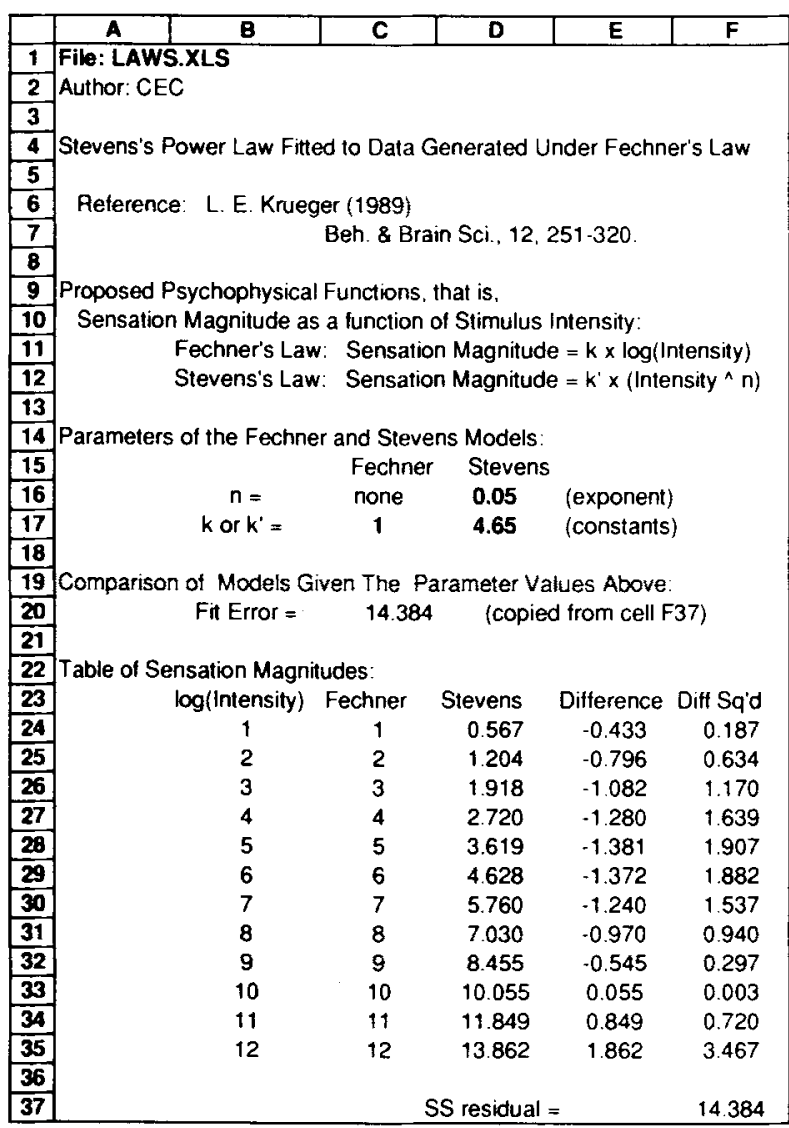

Figure 6. Spreadsheet LAWS.XLS, illustrating a comparison of Stevens's power law and Fechner's law. Entering new parameter values in cells D16 and D17 would cause the predicted values for Stevens's law in cells D24-D35 to be recalculated. Also, entering a new constant in cell $\mathrm{C} 17$ would cause a rescaling of the Fechner predictions in cells C24-C35. The fit error value in cell $\mathrm{C20}$ is a measure of the discrepancy between the two laws for the given parameter values. 


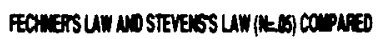

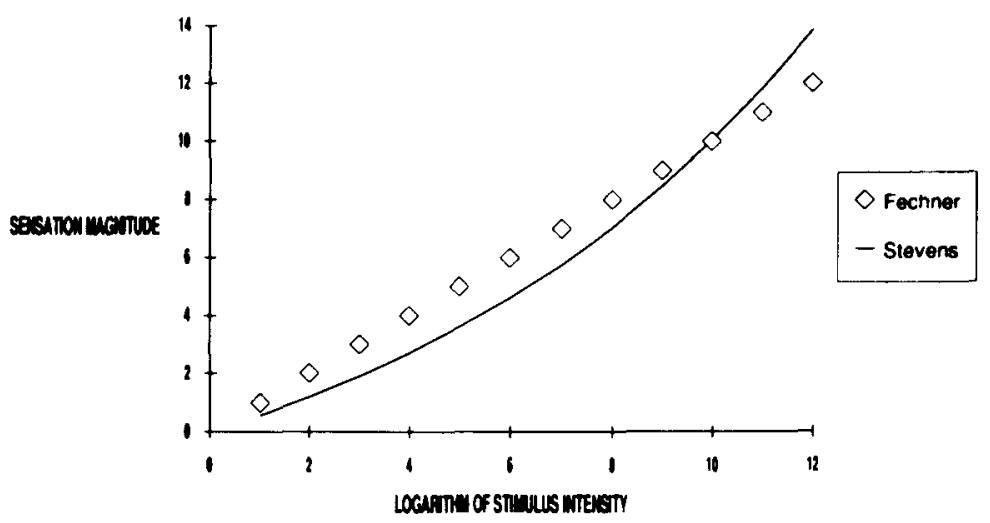

Figure 7. Chart LAWS.XLC, giving a graphical comparison of the Stevens and Fechner functions generated by spreadsheet LAWS.XLS.

of the discrepancy between the Fechner and Stevens values.

Keeping Fechner's law constant by leaving cell $\mathrm{C} 17$ alone, a student could enter different values for the parameters of Stevens's power law in cells D16 and Di7, and observe their effects on the data, the graph, and the goodness-of-fit measure. A good exercise is to try to make the sum of squared differences in cell $\mathrm{C} 20$ as small as possible. Krueger (1989) noted that reducing the exponent (to .001) while increasing the scaling constant (to about 430 ) brings the two laws into even closer agreement. Such a change in the parameters of the spreadsheet improves (reduces) the fit error value to .002 and results in curves that virtually coincide.

\section{Other Models to Try}

Most well-defined models can be implemented with the use of spreadsheets, certainly for purposes of instruction (including self-instruction), but also for some original research work. Here is a short list of sources for potential spreadsheet models that may be of interest to psychologists: (1) other models of Pavlovian conditioning, including those of Hawkins and Kandel (1984), Sutton and Barto (1987), and Gluck and Bower (1988); (2) various aspects of signal detection theory (Green \& Swets, 1966) and other models of detection, recognition, and discrimination; (3) scalar timing theory (e.g., Gibbon, Church, \& Meck, 1984), the principal model of time perception in animals; (4) several models for human time perception, including those of Kristofferson (e.g., 1977) and Creelman (1962), and motor timing (e.g., Wing \& Kristofferson, 1973); (5) simple connectionist models such as Anderson's brain-state-in-a-box (Anderson, 1977) and the algebraic components of many more complex models (e.g., Jordan, 1986); (6) many models for reaction and response times (Luce, 1986); (7) models of chaotic dynamics and fractal properties of data such as EEG records (Rapp et al., 1986).

This list has not been constructed systematically, and it is quite idiosyncratic. However, it should give some indication of the range of models, specifiable by a few equations each, that might be examined in spreadsheet form.

\section{Summary}

The purpose of this article has been to encourage academic psychologists to consider using spreadsheets for teaching and studying models appropriate to their specialty areas. "What-if" modeling has gained acceptance as a useful tool in the business world, but it would seem to have even more applicability in teaching and research. Two attractions of spreadsheets are their potential as an alternative to traditional programming and their value as a display medium for examining the model's behavior, especially when tables are hot-linked to charts. Three examples from psychology have been used to illustrate a few specific spreadsheet modeling techniques. Style suggestions for constructing spreadsheets have been offered, and some other models have been identified as candidates for spreadsheet implementation.

\section{REFERENCES}

ANDerson, J. A. (1977). Neural models with cognitive implications. In D. LaBerge \& S. J. Samuels (Eds.), Basic processes in reading. perception and comprehension (pp. 27-90). Hillsdale, NJ: Erlbaum.

Bjork, R. A. (1973). Why mathematical models? American Psychologist, 28, 426-433.

Cox, T. (1978). Stress. Baltimore: University Park.

Creelman, C. D. (1962). Human discrimination of auditory duration. Joumal of the Acoustical Society of America, 34, 582-593.

EsTes, W. K. (1975). Human behavior in mathematical perspective. American Scientist, 63, 649-655.

FeChNER, G. T. (1966). Elements of psychophysics (H. E. Adler, trans.). New York: Holt. (Original work published 1860)

Gibbon, J., Сhurch, R. M., Meck, W. H. (1984). Scalar timing in memory. In J. Gibbon \& L. G. Allan (Eds.), Timing and time perception (Annals of the New York Academy of Sciences, Vol. 423, pp. 52-77). New York: New York Academy of Sciences.

Gluck, M. A., Bower, G. H. (1988). From conditioning to category learning: An adaptive network model. Joumal of Experimental Psychology: General, 117, 227-247.

Green, D. M., SwETs, J. A. (1966). Signal detection theory and psychophysics. New York: Wiley. 
HALFF, L. A. (1987). A spreadsheet simulation of the early stages of visual information processing. Behavior Research Methods, Instruments, \& Computers, 19, 117-122.

HAWKINS, R. D., KANDEL, E. R. (1984). Is there a cell-biological alphabet for simple forms of learning? Psychological Review, 91, 376-391.

HEWETT, T. T. (1985). Teaching students to model neural circuits and neural networks using an electronic spreadsheet simulator. Behavior Research Methods, Instruments, \& Computers, 17, 339-344.

HEwETT, T. T. (1988). The electronic spreadsheet as a professional productivity tool. Behavior Research Methods, Instruments, \& Computers, 20, 231-235.

JoRDAN, M. I. (1986). An introduction to linear algebra in parallel distributed processing. In D. E. Rumelhart \& J. L. McClelland (Eds.), Parallel distributed processing: Vol. 1. Foundations (pp. 365-422). Cambridge, MA: MIT Press.

Kristofferson, A. B. (1977). A real-time criterion theory of duration discrimination. Perception \& Psychophysics, 21, 105-117.

KRUEGER, L. E. (1989). Reconciling Fechner and Stevens: Toward a unified psychophysical law. Behavioral and Brain Sciences, 12, 251-320.

LAZARUS, R. S. (1976). Patterns of adjustment (3rd ed.). New York: McGraw-Hill.

LUCE, R. D. (1986). Response times: Their role in inferrring elementary mental organization. New York: Oxford University Press.

NevisOn, J. M. (1990). Microsoft Excel spreadsheet design. New York: Brady.
Rapp, P. E., Zimmerman, I. D., Albano, A. M., de Guzman, G. C., Greenbaum, M. N., Bashore, T. R. (1986). Experimental studies of chaotic neural behavior: Cellular activity and electroencephalographic signals. In H. G. Othmer (Ed.), Nonlinear oscillations in biology and chemistry (pp. 175-205). Berlin: Springer-Verlag

Rescorla, R. A., \& Wagner, A. R. (1972). A theory of Pavlovian conditioning: Variations in the effectiveness of reinforcement and nonreinforcement. In A. H. Black \& W. F. Prokasy (Eds.), Classical conditioning II: Current research and theory (pp. 64-99). New York: Appleton-Century-Crofts.

ROGERS, R. L. (1989). Using the microcomputer as a visual aid in the statistics classroom. Behavior Research Methods, Instruments, \& Computers, 21, 96-98.

Stevens, S. S. (1957). On the psychophysical law. Psychological Review, 64, 153-181.

SteVEns, S. S. (1961). To honor Fechner and repeal his law. Science, $133,80-86$.

SUtTon, R. S., \& BARTo, A. G. (1987). A temporal-difference model of classical conditioning. In Proceedings of the Ninth Conference of the Cognitive Science Society (pp. 355-378). Hillsdale, NJ: Erlbaum.

Wing, A. M., \& Kristofferson, A. B. (1973). Response delays and the timing of discrete motor responses. Perception \& Psychophysics, 14, 5-12.

(Manuscript received September 14, 1990; revision accepted for publication February 18, 1992). 